\title{
Electrospun MWCNT/polyaniline composite nanofibers for liquefied petroleum gas (LPG) sensing
}

\author{
Pallavi T. Patil*, Hemlata J. Sharma and Subhash B. Kondawar \\ Department of Physics, Polymer Nanotech Laboratory, RashtrasantTukadojiMaharaj Nagpur \\ University, Nagpur - 440033, India \\ *Email: pallavitpati11986@gmail.com
}

In this paper we report, fabrication of poly(methyl methacrylate) (PMMA)nanofibers by electrospinning technique and used as substrate for coating pure polyaniline (PANI) and $\mathrm{PANI} /$ multiwalled carbon nanotubes (MWCNT) separately during chemical oxidative polymerization (Figure 1) [1,2].

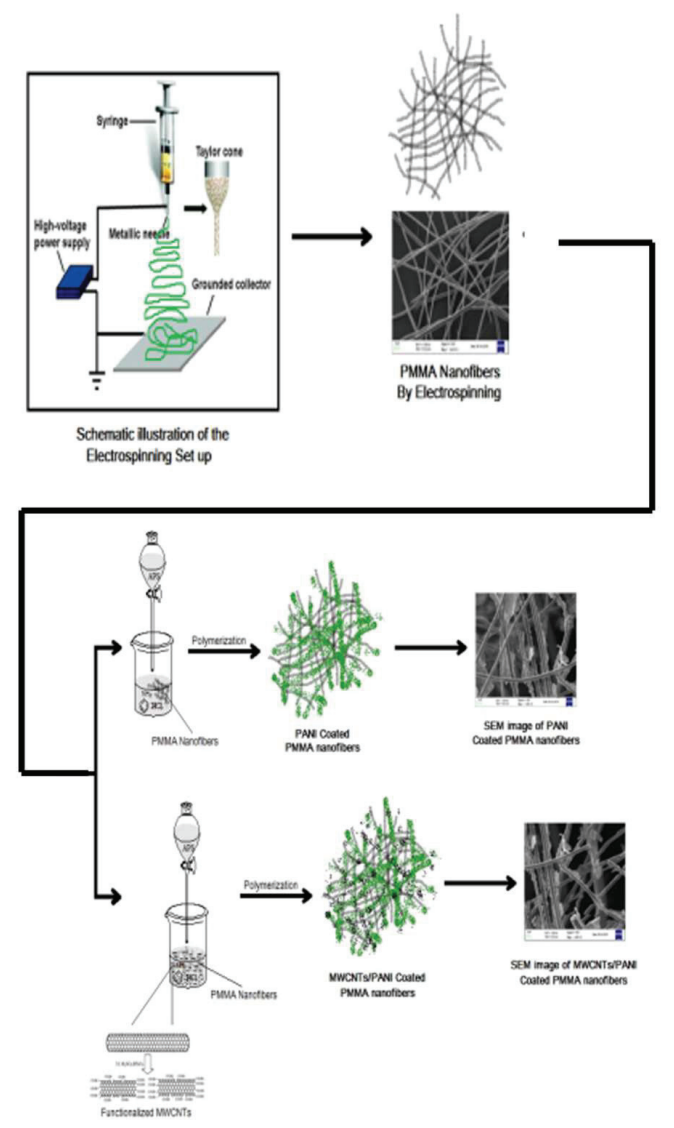

Figure 1: Schematic illustration of synthesis process of the PANI and the MWCNT/PANI on PMMAnanofibers
The morphology and structure of PANI and PANI/MWCNT nanofibers were investigated by SEM, UV-VIS, FTIR and XRD. SEM shows that the fibrous structure with the diameter in the range between 200-300 nm. UV-Vis, FTIR and XRD analyses confirmed the formation of PANI and its interaction with MWCNT. The change in electrical resistance of PANI and PANI/MWCNT for different ppm of liquefied petroleum gas (LPG) was measured. In case of PANI, sensitivity found to be $47.56 \%$ at $32^{\circ} \mathrm{C}$ and in case of PANI/MWCNT same nature was followed but the sensitivity factor found to be $99.57 \%$ at $32^{\circ} \mathrm{C}$. So PANI/MWCNT showed more sensitivity than PANI. PANI/MWCNT nanofibers showed rapid and reversible resistance change upon exposure to LPG as compared to that of PANI nanofibers, which may be due to MWCNT wrapped by conducting PANI results in the formation of a charge transfer complex which increases the protonation of the polyaniline and the transition in the electrical resistance was attributed to a change in the localization length of the composite nanofibers [3].

Keywords:polyaniline, multi-walled carbon nanotubes, poly (methyl methacrylate), electrospinning, LPG sensing

\section{References}

1.ShanzuoJi, Yang Li, Mujie Yang, Sensors and Actuators B 133 (2008) 644-649

2.PandiGajendran and RamiahSaraswathi, Pure Appl. Chem. 80 (2008) 2377-2395

3.Pallavi T. Patil, Rajshri S. Anwane, Subhash B. Kondawar, Procedia Materials Science 10 (2015) 195-204 